\title{
Analysis and Compare of Cardiac Functional Efficiency Level and the Circulatory System for Short, Middle and Long-Distance Runners
}

\author{
Lamyia Yihia Atwan \\ Assist. Lecturer, Al-Ayen University, Thi-Qar, Iraq
}

\begin{abstract}
The aims of this study are knowing of cardiac functional Efficiency level and circulatory system for the short, middle and long -distance runner and knowing the differences of the cardia physical efficiency level and circulatory system for the long-middle and shirt-distance runners. Thus, continuing of the sport training operation may lead to take place some of the physiological changes in body, as the track and field games is definitely distinguished that runners. The athletes may face a various kind of functional changes resulted by various training resilience. While the Heartbeat size increases with the physical efficiency level at the relation time. Thus, we may say that physical efficiency level increases and reflects with the Heartbeat increasing and lowering of the cardiac Heartbeat during relaxation. As the track and field races are dividing athletes into short, middle and long-distance runners. Generally, the athletes participating may face in various games, therefore, the research importance is based on knowing the cardia physical efficiency level and circulatory system for the short, middle long-distance runners, then attempting to know the best set in fitness in raspatory circulatory system among the athletes in these activities.
\end{abstract}

Keywords: Cardiac, Efficiency Level, Circulatory System, Distance Runners.

\section{Introduction}

The physical activity is deeply linking with the vital systems as it being an attempt to developing the individual capacities and progressing his aptitudes in order to achieve a better level in performance as well as creating the functional endurance for the human systems which is directly connected or indirectly with the present activity ${ }^{1}$.

One of the most important systems is circulatory system and respiratory. And the heart will be the main part of the circular system and the Heart activity of muscualr contraction and relaxation in succession ${ }^{2}$. This sucession in Heart activity will ensure the blood reaching to the veins and cardiccirculatory ${ }^{3}$. It will contain contraction and relaxation. And a time of stopping of heart contraction, which is consequence tunes to relaxations of Atriums and Ventricles, and stopping is the rest between the relaxation of Atriums and ventricles. The measures and functional tests are considered one of the most important factors which to accompany the treatment program. In order to ensure resilience training of for the runner and having the increasing resilience, either fix it or lesson it to be able by finding out any shortage unnormal in the normal state for the athlete at the beginning. Before is multiplied during the training process and increasing of resilience degree to the body, without noticing the healthy and functional training state. The means of the test and measure have been developed to include all the data of the athlete during relaxation time and at the physical resilience and later. Ans these tests and measures also provide to follow up the training runner state during the training seasons which makes is a precursor to prophecy of the an indicate do of better future level

The distinguished tests of the functional state for Heart and Circular system: The cardiac efficiency and circulatory system are considered of the important mark for the physical fitness. The cardiac functional and circularity system are based on the ability for this part. And this system in order to fill up the boys needs of 
blood during exercises and ant ability to return back to the natural state after a short period of relaxation

Cardiac functional state and its relation to the physical ability: The term of body efficiency is one of the greatly distributed terms in athletic physiology field and work. The experiments conclude that body efficiency is increased in level and connected with the circulatory system efficiency as the size of the Heartbeat is increased with the body efficiency level and reflected on the Heartbeat size increasing and lesson Heartbeat average during relaxation.

Circulatory system: The circulatory system is divided into two parts: the cardiac circulatory system which included the heart which is the main member and bloody lymph veins, and lymph glands so the heart circulatory system pushes the oxygen and various food material into the body cells. and at the same time attempting to get rid of $\mathrm{CO}_{2}$.

Of $\mathrm{CO}_{2}$ the remainmetapolism processs of the all human cells. It also provides transporting hormones from endocrine glands into its limited cells. This system also keeps the interior body heat degree at roughly (37c) and the ability to keep an amount of the liquids in body in order to save the body out of damage at the dryness state and help of saving from infections by germs and microbes which attack and occupied the body.

\section{Activities of Track and Field Games (Running):}

Activities of Short- Distance: Short-distance running is considered one of the activities which required a great amount of physical and psychological capacities. And the short -distance running is distinguished of being reliable of airless energy system in which the body woks during oxygen shortage. And the short-distance activities are considered of the activities which in excitement is important as it is distinguished of surprises. Thus, these activities are considered totally competing and not-entertainment (1). The short-distance activities are including competitions $(100 \mathrm{~m}, 200 \mathrm{~m}, 400,100$ fences female. 110 fences male, $4100 \mathrm{~m}$ post, $400 \mathrm{~m}$ post)

Activities of middle-distance: The middle- distance running is distinguished in the ongoing time with high acceleration performance level in the last years. The difficulty of trespassing the world numbers in this field is very hard to obtain, in order to reach to a higher level

The middle-distance runners is described of gathering amid the long-distance an short-distance runners (1). It included of the middle activities (800 m. $1500 \mathrm{~m})$

Activities of Long-Distance: These activities are named the long running distances or the endurance running. Thus, the higher rate of the values to ensuing the energy is oxygen rate is amount between (70-80)

The long -distance activities are considered of the simpler distances in the track and field games technically. The athlete can learn by himself as not need to the technical parts in the running performance and organize the respiratory operations with the step tunes. And this is an important operation so the athlete will not feel of tiredness (2) and the long -distance activities of (3000 m, 3000 fences, $5000 \mathrm{~m}, 10000 \mathrm{~m}$ ).

\section{Research Method and its field measures:}

Research Method: The researcher used the description method of scanning style due being the best method and easily done in making the research aims

Research Society: The aims which the researcher may do for his research and the measures he used will repeat the sample nature which he has chosen (1) therefore the research society for the long, middle and short -distance runners as it is seen in the table 1.

Table (1). The total number of the long and short-distance runners shows and the number which has been tested and the percent rates

\begin{tabular}{|l|c|c|c|}
\hline Statistics treatments-samples & Total number & Number which has been chosen & Percent values \\
\hline Short -distance runners & 5 & 5 & $100 \%$ \\
\hline Middle-distance runners & 4 & 4 & $100 \%$ \\
\hline Long-distance runners & 4 & 4 & $100 \%$ \\
\hline
\end{tabular}




\section{Instruments and the ways of data gathering}

* Foreign and Arabic Resources.

* Cardiac functional measure state and circulatory system.

* tests and measure.

\section{Used Tests:}

Tests of functional state measure and circulatory system

Test name: Step test for Basra university.

Reason for test: Cardiac functional state measure and circulatory system

\section{Used instruments}

Stage at 15 inches

Instrument for record sounds

Result registration form

Method of performance: The student stands in the front of the device while he is holding the crossbar of the device .when the starting signal is given,the expert will climb on the right foot and then the left foot and then the right foot and then the left foot go down and the process of going up and down the foot is done according to the sound rhythm specified for that bump thus the performance continues until the end of time which is 3 minutes.

\section{Registration:}

Calculating performance seconds numbers

Calculating Heartbeat for 30 minutes period after giving the player a rest period for 30 minutes

\section{Evaluation:}

Player's activity is evaluated according to following equation

\section{Player's Efficiency: performance seconds number $\times 100$}

\section{$6.5 \times$ Heartbeat for 30 seconds}

Exploratory Experience: The researchers conducted a exploratory experiment on $2 \backslash 7 \backslash 2020$ on a sample of track and field runners for young men. The aim of this experiment was to identify the efficiency of devices and tools, as well as knowing the work obstacles and the time needed for testing, and the exploratory experiment has achieved its goals.

Main Experience: After making sure of the efficiency of the devices and tools, the players were notified of the test dates. Also, it has been prepared the devices and tools, and the assistant staff were also informed. Later the study experiment has been carried out on 17/2/2020.

Statistical means: The test results have been statistically treated by using the statistical program (spss)version 20 and by using computer, Lenovo, with speed processor $2,8 \mathrm{~Gb}$. according to following rules:

Percentage

analyzing parallel variance (ON WAY ANOVA)

L.S.D rule

Arithmetic mean

Standard deviation

Showing experiment, analyze and discuss

Showing, analyzing, and discussing the test results of cardiac functional state and circulatory system for the short, middle, long distance runners.

Table (2). Showing the Arithmetic Mean and Standard deviation the test results of cardiac functional state and circulatory system for the short, middle, long distance runners.

\begin{tabular}{|l|c|c|}
\hline Statisticslthe sample & s & \\
\hline Short distance players & 91,13 & 3,6 \\
\hline Medium distance players & 2,8 & 75,89 \\
\hline Long distance players & 3,86 & 94,78 \\
\hline
\end{tabular}

- From table 2 we find that arithmetic mean for testing the cardiac functional state and circulatory system for short- distance players which reached to $(91,13)$ with standard deviation reached to $(3,6)$. While the arithmetic mean for short- distance runners $(75,89)$ with standard deviation $(2,8)$. While the arithmetic mean for the long-distance runners reached to $(94,78)$ with $(3,87)$. Therefore, we find out the variation in the level of Cardiac functional and the circulatory system between the short middle and 
long- distance runners. And in order to identify these differences, the researcher carried out an analysis (on way a nova) and it shows in the following table:

Table (3): Data analysis shows the level of the cardiac functional state and the circulatory system at Short, middle and long distances

\begin{tabular}{|l|c|c|c|c|c|}
\hline Variation resources & Cubes total & Free-work degree & Free-work degree & Cube average & Table value (f) \\
\cline { 1 - 5 } Of groups & 817,065 & 2 & 408,532 & \multirow{2}{*}{5,527} & \multirow{2}{*}{4,1} \\
\cline { 1 - 2 } Inside groups & 739,209 & 10 & 73,921 & & \\
\hline Total & 1556.418 & 12 & 482.453 & & \\
\hline
\end{tabular}

By knowing table 3 we find out the calculated value of $F$ which reached to $(5,527)$ and this value its bigger than value tablet adults $(4,10)$ under degree freedom $(10,20)$. Thus, there will be nominal differences amid the short, middle and long-distance runners in testing the cardiac state and circulatory system. For this, in order to learn if there any effect which may record for the sake of the runners in these three activities, the researcher has carried out an operation to find out the less nominal value (L. S. D). and for knowing on the best group in this test, as the less nominal value using the best group in it, just to learn the best performance, there has to be a difference in the results of difference analyzation. In this way, (L. $\mathrm{S} . \mathrm{D})$ is calculated when there will be differences in the value of (F) more of calculated (F)

Table 4: It represents the less nominal difference value and the differences for the average to the short, middle and long-distance runners:

\begin{tabular}{|l|c|c|c|c|}
\hline Statistics/samples & Short & Middle & Long & Calculated value at L.S.D \\
\hline Short-distance runners & & 15,24 & 3,65 & \multirow{2}{*}{13,03} \\
\hline Middle-distance runners & & & 18,89 & \\
\hline Long-distance runners & & & & \\
\hline
\end{tabular}

By seeing table 4 we can find out the difference amid the calculated media amid the long and short and middle-distance runner which reached to $(15,240$. This difference amid the medias is the larger of value and calculated (L.D.S) this concluded of presenting a nominal difference in selecting the cardiac functional level and circulatory system for the sake of the middle -distance runners due the calculated media for the middle -distance runners is the lessor value than the cleated media for the

While the difference of the calculated media between the short and long-distance runners may reach to $(18,89)$ and the difference between the calculated media value are larger than the calculated value of (L.S.D). That leads to find out a difference in choosing the Cardiac Functional State and Circulatory System for the sake of the short-distance runner.
The researcher may explain the reason that makes the used test distinguished in what accelerated performance and to the continuous effort in performance which leads the middle-distance runners to better than he long and short-distance in this test.

The researcher may explain the reason that makes the middle-distance runner of having a large lynx ability and for distinguishing of having larger cardiac muscle size which reached to average of ability $(1200 \mathrm{~cm} 3)$ also to the pulmometry size which reached to average (47005300) (1).

The middle-distance running distinguishes in the present time with huge acceleration. Thus, the middledistance runners need to acquiring a natural special talent due the highly required performance in the last years. Therefore, we may find out of highly training programs which serve of developing the psychological, physical 
and bodily qualities. And some of the middle-distance runner may be described of gathering performance between the long distance and short -distance runners.

\section{Conclusions and Recommendations}

1. Cardiac functional Efficiency Level and circulatory System for the middle -distance runners are in better Functional Efficiency Level for the Short and Long distance runners.

2. There will be no difference in functional efficiency level in testing the cardiac functional state and circulatory system amid long and short- distance runners

Financial Disclosure: There is no financial disclosure.

Conflict of Interest: None to declare.

Ethical Clearance: All experimental protocols were approved under the Al-Ayen University and all experiments were carried out in accordance with approved guidelines.

\section{References}

1. Amerallah Ahmed al-Basati: the foundations and rules of sports training and its applications, Aleskendiya, Munshat al-maaref. 1988.

2. Raed Muhammad Mashtat et al, technicizing cardiac functional state measure test and circulatory system. Research published, Babylon journal of humanities number 4511,2006.

3. Raisankhraibit Majeed: Scientific Research Method in physical education. Al-Mosul, directorate of Dar al-Kutoob for printing and distribution, 1987.
4. Sati Ismael: traning Effect under air pressure various on the functional adaptation and performance for the long-distance runners in Iraq, doctorate Thesis, University of Baghdad, sport education college, 2003.

5. Sulaiman Ali Hassan, Awatif Ahmed Labeeb, Progressing the muscular power. First publishing, Dar al-Fikri al-Arabi, 1987.

6. Qasim Hassan Hussein, Mandsoor Jameel, Mohammed Uthman, Athlete games encyclopedia, Dar al-Qalam for publishing and distribution, fist publishing University of Baghdad, Ministry of Higher education and scientific research, 1998.

7. Qasim Hassan Hussein, Mansoor Jameel,: Physical fitness and the ways to achieve, University of Baghdad, Ministry of Higher education and scientific research, 1988.

8. Kadhim Jabir Amir: Physiological measures and test in sport field. First publishing. Al-Kuwait, 1997.

9. Mohammed Hassan Alawi, Abul-Ila Ahmed Abid al-Fatah physiology of sport training. Cairo, Dar alFiker al-Arabi, 2000.

10. Mohammed Othman: Athletic games encyclopedia, Dar al-Qalam for publishing and distribution. 1990.

11. Marwan Abdulmajeed Ibrahim: descriptive and refrcnesttists. Fist publishing, Uman, Dar-Alfiker for printinnd publishing. 2000.

12. Nahida Abdul-Zahra al-Asadi: Measure of some fields as precursor of athletic cacti for short, middle and long-distance runners. Doctorate thesis. Sport education college, Basra university, 2004. 